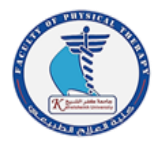

\title{
Effect of sensory integration on gait for children with mild hemiplegic cerebral palsy
}

\author{
Mohamed K Seyam ${ }^{1 *}$, Abdel Aziz A Sherief ${ }^{2}$, Mohamed I Waly ${ }^{3}$, Faizan Z Kashoo', \\ Mahmoud S. Elfakharany ${ }^{4}$
}

${ }^{1}$ Department of physical therapy and health rehabilitation, College of applied medical sciences, Majmaah University, Al Majmaah 11952 - Saudi Arabia.

${ }^{2}$ Department of physical therapy for growth and developmental disorders in children and its surgery, Faculty of physical therapy, Kafer El Sheikh University, Egypt.

${ }^{3}$ Department of medical equipment technology, College of applied medical sciences, Majmaah University, Al Majmaah 11952 - Saudi Arabia.

${ }^{4}$ Department of physical therapy for Paediatrics, Faculty of Physical Therapy, Cairo University. Egypt.

\begin{abstract}
*Correspondence to Mohamed K. Seyam Department of physical therapy and health rehabilitation, College of applied medical sciences, Majmaah University, KSA.

Email:

m.seyam@mu.edu.sa
\end{abstract}

Published online: Sept 2021

\begin{abstract}
:
Background: Children with hemiplegic cerebral palsy exhibit increased muscular tonicity causing impaired postural control. Postural control is essential to maintain posture and balance during bipedal walking. The purpose of this study was to examine the efficacy of the sensory integration on gait parameters among children with hemiplegic cerebral palsy.

Methods: Thirty boys with hemiplegic cerebral palsy with ages ranged from 10 to 14 years participated in this study. They were randomly assigned into two groups. The control group (A) received a conventional physical therapy program while the experimental group (B) received the conventional physical therapy program and the addition of sensory integration. Both groups received treatment sessions five times per week for three successive months. Kinematic gait parameters were evaluated before and after three successive months of treatment.

Results: The non-parametric analysis (Wilcoxon Signed Ranks Test) of data revealed non-significant differences at baseline (between-groups). However, statically significant improvement $(\mathrm{p}<0.5)$ was seen in both groups (within-group). Further, a statistically significant difference (between-group) $(\mathrm{p}<0.5)$ was observed between post-treatment values of gait.

Conclusion: Sensory integration along with a conventional physical therapy program for 3 months led to the statistically significant difference in the gait parameters among children with hemiplegic cerebral palsy.

Key words: Cerebral palsy, Children, Gait, Sensory integration therapy.
\end{abstract}

\section{Introduction}

Cerebral palsy (CP) is defined as a group of nonprogressive motor impairment resulting from a lesion in the developing brain, affecting movement and posture. $\mathrm{CP}$ children vary in clinical presentation, type of lesion, severity, and extend of impairment. There are disturbances in sensation, cognition, communication and perception (1).CP are classified according to the type of lesion in the brain, signs, and symptoms (Spastic, ataxic, dyskinetic \& mixed CP), time of insult (Prenatal, intra-natal, postnatal), Topographical involvement of limbs (Hemiplegia, diplegia, or 
quadriplegia), change in muscle tone (Hypotonic, isotonic, hypertonic) (2). The prevalence of $\mathrm{CP}$ is between 2-2.5 per 1000 live birth (3). Sensory integration therapy (SIT) is an intervention aiming to improve the life quality of persons by improving sensory experiences (4). SIT is an active therapy that incorporates sensory bombardment through various tools to improve children's sensory experience. SIT gives the nervous system the chance to change "Neuroplasticity" and then provides the correct dosage of sensory stimulation resulting in impairing the ability of the nervous system to process the sensory stimulus(5). Subsequently, the behavior problems will be reduced and the efficacy of learning will be increased (6). SIT uses large pieces of tools like balls, trampolines, rolls, and swinging hammocks. They help in improve proprioception, tactile and vestibular practices $(7,8)$. The central and peripheral nervous systems managing the incoming sensory information by the process called sensory processing, This processing component are reception, integration, modulation, organization, of sensory stimuli as well as sensory input behavioral responses, Although the term sensory integration is similar to the sensory processing term, the two terms aren't interchangeable; sensory integration is the only one component of the more expansive term "Sensory processing" (9). Previous hemiplegic cerebral palsy (HCP) gait studies have focused primarily on deviations from the affected side during various phases (10-13). The differences are associated with many causes such as tightness of the muscle, reduced movement ability, bony deformity, and impaired motor function. The purpose of our study is to investigate the efficacy of the sensory integration intervention on spatial and temporal gait parameters among children with hemiplegic cerebral palsy.

\section{Subjects, randomization and methods}

\subsection{Subjects}

Thirty boys with spastic hemiplegic CP with ages ranged from 10 to 14 years were selected from Outpatient Clinic, Faculty of Physical Therapy, Kafr ElSheikh University. The inclusion criteria for the selection of the participants include; children diagnosed with spastic hemiplegia. They had stable medical and clinical status. The children were able to walk 10 meters independently. The participants were able to understand and follow simple instructions. Exclusion criteria include any sensory impairment or psychological disorders. The Children who had any congenital or acquired skeletal deformities as metatarsus adductus, internal or external tibial torsion, femoral anteversion or retroversion, genu varum or valgum, and pes planus were excluded (14) Also, we excluded the participant who had undergone recent orthopedic surgical intervention.

The children were allocated into two equal groups randomly. The control group (A) and the study group (B). We explained the aims, purpose, and steps of the study to the participant's children and their parents or guardians. Also, the potential risks and benefits were explained to the children and their parents. We followed the Declaration of Helsinki and the ethics code of the world medical association for experiments for humans. The protocol was submitted to the Institutional Review Board of the Cairo University, and ethical approval was obtained. Participants were provided an informed written consent form before starting the study. Parents of the children or children's guardian signed a consent form before participation.

\subsection{Randomization}

We assessed thirty-six children for the eligibility criterion. We excluded four children as they did not meet the inclusion criteria, and two children were excluded as their parents refused to participate in the study. After we had taken the baseline measurements, the randomization process was applied by closed envelopes. The researcher prepared 15 closed envelopes labeled with a group (A)and another 15 closed envelopes labeled with a group (B). Then, we asked each participant to draw a closed envelope that contained one of the two groups.

\subsection{Methods}

\subsubsection{Pre-intervention evaluation}

We assessed the gait parameters for participant children were before the treatment and after three months of treatment. We used the Biodex gait trainer 2TM. We gave one oration session for children to be familiar and comfortable with the equipment, the research team, and protocol.

\subsubsection{Biodex gait trainer 2TM}

In the beginning, the children were oriented to the equipment before calculating the gait parameters. Before the actual assessment was recorded, the children had asked to perform preparatory walking on the treadmill. The children wore a safety belt around his pelvis throughout the session. The examiner was standing near to the children while the children performed walking. The starting speed of the equipment was 0.3 meters per hour. Then gradually we increase the 
speed of the Biodex gait trainer to reach a comfortable pace for the participant. After the child becomes familiar with the routine the gait parameters were recorded in the middle of the session without informing the participant. We allowed each participant to perform walking for three minutes continuously. The ending of the assessment session was done by gradually decrease the speed of the treadmill until stopped and remove the safety belt from the child. The result was shown on the Biodex gait trainer screen. The total of the threeassessment session was done for each child with a rest period in-between, and the average of each gait parameter in the three evaluations was recorded. The gait parameters include average gait cycle, average step length, and step symmetry (right leg to left leg time distribution).

\subsubsection{Treatment sessions}

Control Group (A): All participants in group A performed a conventional physical therapy exercise program. The frequency of exercises was 5 sessions per week for three successive months. The type of exercises includes exercise for stretching the tight muscles, exercises on wobble board for the facilitation of equilibrium and righting reactions, exercises for strengthening the weak muscles, open and closed surroundings gait training, and training program on the treadmill. The mode of exercise started with warming up for 3-5 minutes, then the main program start at the end the cool-down was done for 3-5 minutes.

Study Group (B): All participants in group B received an exercise program (tailor-made) in addition to sensory integration therapy. Each treatment session includes the same tailor-made exercise program that is done for group $\mathrm{A}$ in addition to components of sensory integration treatment. The sensory integration program includes 10 minutes of walking in the water pool (level of water till the waist level) 10 minutes walking on sand and throwing and catching a rubber ball for 10 minutes.

\section{Statistical analysis}

The collected data of the gait parameters of control group A and group B were statistically analyzed to compare the effects of the sensory integration intervention on gait parameters among children with hemiplegic cerebral palsy. Descriptive statistics were done in the form of the mean and standard deviation. Due to the non-normal distribution of data and small sample size, the Wilcoxon Signed Ranks Test was conducted for comparing the pre and post-treatment mean values of all measuring variables between both groups. Mann-Whitney U was conducted for comparing pre and post-treatment mean values in each group. All statistical analyses were conducted through SPSS (statistical package for social sciences, version 25).

\section{Results}

Our results showed that there was no significant difference between the two groups at the baseline assessment $(P$-value $>0.05)$. The comparing results of the gait parameters within groups were showed in table $1,2 \& 3$. The results of the mean values of the average step length, time in each foot, and an average of the gait cycle of the pre and post physical therapy intervention appeared that there is a significant difference within groups as $P$ value $<0.05$. (Table $1,2,3$ )

Table1: The intra-groups comparison between before and after physical therapy treatment means values of average step length for control group \&study group.

\begin{tabular}{|c|c|c|c|c|c|c|c|c|}
\hline Group & \multicolumn{4}{|c|}{ Control group A } & \multicolumn{4}{|c|}{ Study group B } \\
\hline \multirow{2}{*}{ Variable } & \multicolumn{2}{|c|}{ Rt. Foot } & \multicolumn{2}{|c|}{ Lt. foot } & \multicolumn{2}{|c|}{ Rt. foot } & \multicolumn{2}{|c|}{ Lt. foot } \\
\hline & Pre & Post & Pre & Post & Pre & Post & Pre & Post \\
\hline $\mathrm{X}$ & 0.386 & 0.425 & 0.392 & 0.432 & 0.387 & 0.443 & 0.393 & 0.452 \\
\hline SD & 0.006 & 0.016 & 0.014 & 0.014 & 0.018 & 0.035 & 0.027 & 0.034 \\
\hline Z & \multicolumn{2}{|c|}{-3.436} & \multicolumn{2}{|c|}{-3.249} & \multicolumn{2}{|c|}{-3.28} & \multicolumn{2}{|c|}{-3.243} \\
\hline$P$-value & \multicolumn{2}{|c|}{0.0005} & \multicolumn{2}{|c|}{0.001} & \multicolumn{2}{|c|}{0.001} & \multicolumn{2}{|c|}{0.0001} \\
\hline Significant & \multicolumn{2}{|c|}{$*$} & \multicolumn{2}{|c|}{ * } & \multicolumn{2}{|c|}{ * } & \multicolumn{2}{|c|}{ * } \\
\hline
\end{tabular}

Rt.: right, Lt.: left, X: mean, SD: stander deviation, Z: Wilcoxon Signed Ranks Test, $P$-value: level of significance, *: Significant at $P<0.05$.

Table2: The intra-groups comparison between before and after physical therapy treatment means values of time of Rt. \&Lt. foot for control group \&study group.

\begin{tabular}{|c|c|c|c|c|c|c|c|c|}
\hline Group & \multicolumn{4}{|c|}{ Control Group } & \multicolumn{4}{|c|}{ Study Group } \\
\hline \multirow[t]{2}{*}{ Variable } & \multicolumn{2}{|c|}{ Rt. Foot } & \multicolumn{2}{|c|}{ Lt. foot } & \multicolumn{2}{|c|}{ Rt. Foot } & \multicolumn{2}{|c|}{ Lt. foot } \\
\hline & Pre & Post & Pre & Post & Pre & Post & Pre & Post \\
\hline $\mathrm{X}$ & 41.3 & 41.2 & 41.8 & 41.2 & 44.7 & 45.1 & 48.5 & 47.9 \\
\hline SD & 1.3 & 1.8 & 1.4 & 1.6 & 1.5 & 1.2 & 1.4 & 2.5 \\
\hline $\mathrm{Z}$ & \multicolumn{2}{|c|}{-3.457} & \multicolumn{2}{|c|}{-3.421} & \multicolumn{2}{|c|}{-3.428} & \multicolumn{2}{|c|}{-3.424} \\
\hline$P$-value & \multicolumn{2}{|c|}{0.0005} & \multicolumn{2}{|c|}{0.0006} & \multicolumn{2}{|c|}{0.0006} & \multicolumn{2}{|c|}{0.0006} \\
\hline Significant & \multicolumn{2}{|c|}{ * } & \multicolumn{2}{|c|}{ * } & \multicolumn{2}{|c|}{ * } & \multicolumn{2}{|c|}{$*$} \\
\hline \multicolumn{9}{|c|}{$\begin{array}{l}\text { Rt.: right, Lt. left, } \mathrm{X} \text { : mean, SD: stander deviation, Z: } \\
\text { Wilcoxon Signed Ranks Test, } P \text {-value: level of } \\
\text { significance, *: Significant at } P<0.05 \text {. }\end{array}$} \\
\hline
\end{tabular}


Table3: The intra-groups comparison between before and after physical therapy treatment means values of average gait cycle for control group \&study group.

\begin{tabular}{ccccc}
\hline & \multicolumn{2}{c}{ Control Group } & \multicolumn{2}{c}{ Study Group } \\
\cline { 2 - 5 } X & Pre & Post & Pre & Post \\
\cline { 2 - 5 } & 0.695 & 0.695 & 0.745 & 0.799 \\
SD & 0.010 & 0.010 & 0.012 & 0.009 \\
Z & \multicolumn{2}{c}{-3.53} & -3.573 \\
P-value & \multicolumn{2}{c}{0.0004} & \multicolumn{2}{c}{0.0003} \\
Significant & \multicolumn{2}{c}{$*$} & \multicolumn{2}{c}{$*$}
\end{tabular}

X: mean, SD: stander deviation, Z: Wilcoxon Signed Ranks Test, $P$-value: level of significance, *: Significant at $P<0.05$.

The statistical analysis of the mean values within the group between the post-treatment mean values of group A with the post-treatment results of group B showed a significant difference between the inter-group results where $P$-value $<0.05$ as (Fig. $1 \& 2$ )

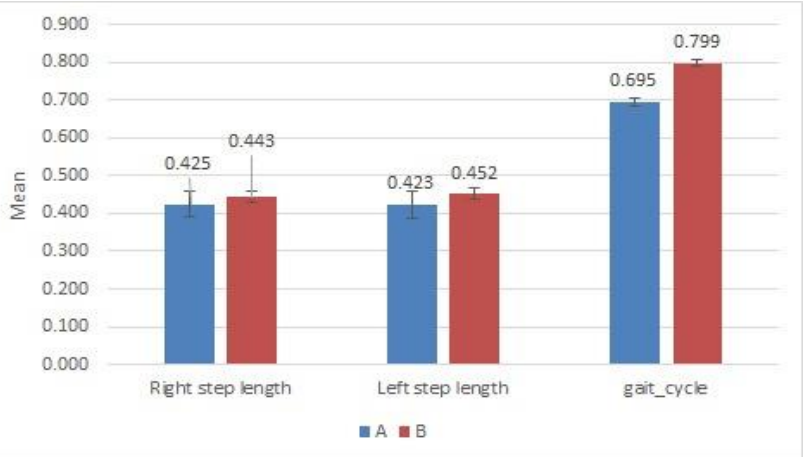

Figure 1: The inter-groups comparison between post physical therapy treatment means values of average step length, and average gait cycle for control group and study group (post-mean values of group A with postmean values of group B).

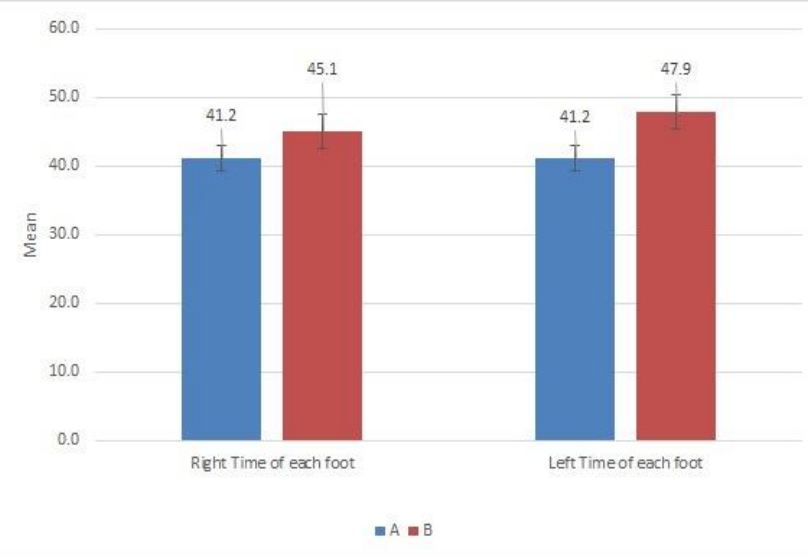

Figure 2: The inter-groups comparison between post physical therapy treatments means values of time of Rt. \&Lt. foot for control group \&study group (postmean values of group A with post-mean values of group B).

\section{Discussion}

The finding of our study showed that children with spastic CP had significantly improved in the temporal and spatial parameters of gait after the addition of SIT. The basic idea of SIT is to provide a rich sensory experience to the children and encourage a meaningful motor output. The use of different sensory information is important because various types of balance disturbances stimulate different sensors and the range of sensitivity of each sensor often varies. If one or more of the systems provide the CNS with misleading information, inputs from the other systems can compensate for this $(14,15)$. Sensory integration allows children suffering from $\mathrm{CP}$ to achieve their maximum sensory and motor function level (16). This approach tries to facilitate normal development and enhances the ability of children to process and integrate sensory information (visual, auditory, proprioceptive, and perceptual) (17). SIT helps to overcome problems in the absorption and processing of sensory information that many young children experience. Ultimately, encouraging such abilities leads to balance and steady motion improvement by training (18). Sensory information about body position and movement (19). For control of posture and movement comes from somatosensory, vestibular, and visual systems $(20,21)$. This information helps CNS to anticipate the forces applied to the body and then generate sufficient muscular action for maintaining posture $(22,23)$. However, there are certain resolutions and importance for each sensory information and the reliability of one type of sensory information can affect another (24). Vestibular stimulation activates healthy postural responses through changes in the sensitivity of the somatosensory and visual systems, suggesting sensory integration (25). There is a method for improving the dynamic balance as well as the ability of postural control called Body awareness training (BAT). BAT consists of repeated simple movements that maintain ability by using limits of stability (26). BAT creates body awareness and allows body awareness to be concentrated on during motion. Movements should be performed in different positions to identify the four body centerline. The centerline of BAT leads to improved balance, postural control, coordination, and free respiration $(27,28)$. Visual-motor integration or Visual-motor coordination (VMC) is the synchronization of visual perceptual skills and fine motor control requires accurate, efficient contact 
between the hand and the eye movements. It combines "Both motor and visual capabilities with the context of environment achieve a target $(29,30)$. The research reported a significant improvement in functionality through body awareness training (BAT) effects on visuospatial neglecting 'mild degree' in patients after acute stroke. Twelve patients were assigned randomly to the control and experimental group which is undergoing BAT for fifteen minutes and the taskoriented training for thirty minutes. The following study indicated that BAT with task-oriented training was feasible and suitable for patients suffering from mild visuospatial neglection in patients after acute stroke (31). This study also supported the findings of HyukShin Cho et.al. (2016) who conducted a study to investigate the impact of BAT on walking and balance ability in patients with chronic stroke. Subjects were allocated randomly to seventeen control and experimental groups which underwent BAT for twenty minutes then walking training for thirty minutes for five days for four weeks and the result suggested that BAT affects balance in chronic stroke patients positively (32). R.C. Miall et.al. in 2001 carried out a test using the human brain's functional magnetic resonance imaging (fMRI) during visually guided tracking tasks requiring varying degrees of eye-hand coordination. During the independent time, the cerebellum was more active than the coordinated eye and hand tracking. Coordination of the engines relies on predictive movement information(33).

\section{Conclusion}

The present study revealed that sensory integration therapy had significantly improving gait pattern for Children with hemiplegic cerebral palsy. Therefore, we recommended physical therapists to add sensory integration therapy for their program in the treatment of children with hemiplegic cerebral palsy.

Data availability: all data for statistical analysis are available if needed.

Conflict of Interest: The authors stated that there is no conflict of interest in this study.

\section{References}

1. Rosenbaum P, Paneth N, Leviton A, Goldstein M, Bax M, Damiano D, Dan B, Jacobsson B. A report: the definition and classification of cerebral palsy April 2006. Dev Med Child Neurol Suppl. 2007;109(suppl 109):8-14.

2. Sanger TD, Delgado MR, Gaebler-Spira D, Hallett M, Mink JW. Classification and definition of disorders causing hypertonia in childhood. Pediatrics. 2003;111(1):e89-97.

3. Stanley FJ, Blair E, Alberman E. Cerebral palsies: epidemiology and causal pathways. Cambridge University Press; 2000.

4. Emmerentia du Plessis. Sensory stimulation therapy, www.academica.edu.

5. Afza F, Manzoor S, Afzal A. How the Development of Tone and Posture Occured in New Borns. 2017;

6. Lang R, O'Reilly M, Healy O, Rispoli M, Lydon H, Streusand W, Davis T, Kang S, Sigafoos J, Lancioni G. Sensory integration therapy for autism spectrum disorders: A systematic review. Res Autism Spectr Disord. 2012;6(3):1004-18.

7. Parham LD, Mailloux Z. Sensory integration. Occup Ther Child. 2005; 5:356-409.

8. Kayihan Hulya GB. Effectiveness of two different sensory-integration programmes for children with spastic diplegic cerebral palsy. Disabil Rehabil. 2001;23(9):394-9.

9. Schaaf RC, Davies PL. Evolution of the sensory integration frame of reference. Am J Occup Ther. 2010;64(3):363-7.

10. Romkes J, Brunner R. Comparison of a dynamic and a hinged ankle-foot orthosis by gait analysis in patients with hemiplegic cerebral palsy. Gait Posture. 2002;15(1):18-24.

11. Armand S, Decoulon G, Bonnefoy-Mazure A. Gait analysis in children with cerebral palsy. EFORT open Rev. 2016;1(12):448-60.

12. Kashoo FZ, Ahmad M. Effect of sensory integration on attention span among children with infantile hemiplegia. Int $\mathrm{J}$ Health Sci (Qassim). 2019;13(3).

13. Romkes J, Hell AK, Brunner R. Changes in muscle activity in children with hemiplegic cerebral palsy while walking with and without ankle-foot orthoses. Gait Posture. 2006;24(4):467-74.

14. Rerucha CM, Dickison C, Baird DC. Lower extremity abnormalities in children. Am Fam Physician. 2017;96(4):226-33.

15. Liu KP, Chan CC, Lee TM, Hui-Chan CW. Mental imagery for promoting relearning for people after stroke: a randomized controlled trial. Arch Phys Med Rehabil. 2004;85(9):1403-8.

16. Ayres AJ, Network PT. Sensory integration and the child: 25th anniversay edition. Revised and updated by Pediatric Therapy Network. Los Angeles, CA: Western; 2005. 
17. Mayston MJ. People with cerebral palsy: effects of and perspectives for therapy. Neural Plast. $2001 ; 8$.

18. Paul S, Sinen P, Johnson J, Latshaw C, Newton J, Nelson A, Powers R. The effects of a sensory motor activities protocol based on the theory of sensory integration on children diagnosed with preprimary impairments. Occup Ther Heal Care. 2003;17(2):19-34.

19. Mochizuki L, Amadio AC. As informações sensoriais para o controle postural. Fisioter Mov. 2006;19(2):11-8.

20. Christovão TCL. Equilíbrio e palmilhas posturais em crianças com paralisia cerebral: estudo clínico aleatorizado controlado. 2013.

21. Kashoo F, Ahmad M. Challenge is the key to stroke rehabilitation. 2018;29(21):3790-1.

22. Faquin BS, Candido CRC, Mochizuki L, Okazaki VHA. Effect of visual and vestibular information on spatial perception on gait. Hum Mov. 2018;19(2):39-45.

23. Kashoo F, Baradie R. Motorized multidirectional protuberance device for sitting balance of stroke patients. In: international journal of stroke. wiley-blackwell 111 river st, hoboken 070305774, nj usa; 2015. p. 89.

24. Kashoo FZ, Ahmad M. Comment to "The effects of Kinesio taping of lower limbs on functional mobility, spasticity, and range of motion of children with spastic cerebral palsy" by Mirjavad Tabatabaee et al. Egypt J Neurol Psychiatry Neurosurg. 2020;56(1):1-2.

25. Horak FB, Macpherson JM. Postural orientation and equilibrium. Compr Physiol. 2010;255-92.

26. Bacsi AM, Colebatch JG. Evidence for reflex and perceptual vestibular contributions to postural control. Exp brain Res. 2005;160(1):22-8.

27. Lindvall MA, Forsberg A. Body awareness therapy in persons with stroke: a pilot randomized controlled trial. Clin Rehabil. 2014;28(12):1180-8.

28. Alharbi RA, Aloyuni SA, Kashoo F, Waly MI, Singh H, Ahmad M. Does transcranial direct current stimulation affect selective visual attention in children with left-sided infantile hemiplegia? A randomized, controlled pilot study. Brain Impair [Internet]. 2020 Dec 10 [cited 2020 Dec 11];1-13.

29. O’Sullivan SB, Schmitz TJ, Fulk GG. Physical Rehabilitation 6th ed (p. 661). Philadelphia, PA FA Davis. 2014.

30. Bang D-H, So Y-J, Cho H-S. Imagery training effects of Upper limb function and Activities of daily living in Subacute stroke patients. J Digit Converg. 2013;11(8):235-42.

31. Bang D-H, Noh H-J, Cho H-S. Effects of body awareness training on mild visuospatial neglect in patients with acute stroke: a pilot randomized controlled trial. J Phys Ther Sci. 2015;27(4):1191-3.

32. Miall RC, Reckess GZ, Imamizu H. The cerebellum coordinates eye and hand tracking movements. Nat Neurosci. 2001;4(6):638-44.

33. Semple RJ, Lee J, Rosa D, Miller LF. A Randomized Trial of Mindfulness-Based Cognitive Therapy for Children: Promoting Mindful Attention to Enhance Social-Emotional Resiliency in Children. J Child Fam Stud [Internet]. 2010;19(2):218-29. Available from: https://doi.org/10.1007/s 